\title{
Vers une approche culturelle du patrimoine forestier : le modèle britannique
}

Towards a cultural approach of forest heritage: the British model

Nach einer kulturellen Betrachtung des Walderbes : das britische Vorbild

\section{Xavier Rochel}

\section{(2) OpenEdition}

Journals

Édition électronique

URL : http://journals.openedition.org/rge/1077

DOI : $10.4000 /$ rge. 1077

ISSN : $2108-6478$

Éditeur

Association des géographes de l'Est

Édition imprimée

Date de publication : 1 janvier 2008

ISSN : 0035-3213

\section{Référence électronique}

Xavier Rochel, "Vers une approche culturelle du patrimoine forestier : le modèle britannique ", Revue Géographique de l'Est [En ligne], vol. 48 / 1-2 | 2008, mis en ligne le 08 octobre 2011, consulté le 08 septembre 2020. URL : http://journals.openedition.org/rge/1077 ; DOI : https://doi.org/10.4000/rge. 1077

Ce document a été généré automatiquement le 8 septembre 2020

Tous droits réservés 


\title{
Vers une approche culturelle du patrimoine forestier : le modèle britannique
}

\author{
Towards a cultural approach of forest heritage: the British model \\ Nach einer kulturellen Betrachtung des Walderbes : das britische Vorbild
}

Xavier Rochel

L'auteur remercie Colin Leslie, de la Forestry Commission Inverness pour les précisions apportées au sujet du projet de restauration à Glenmore.

1 Les forêts européennes sont lourdement marquées du sceau de la «foresterie classique », principalement influencée par les idées allemandes des XVIIIe et XIXe siècles, et dont l'objectif essentiel, pour ne pas dire unique, était la production intensive de bois d'œuvre et d'industrie. Les futaies monospécifiques, et surtout les plantations résineuses héritées de cette vision productiviste de la forêt sont aujourd'hui encore en expansion sur le continent. Pourtant, elles sont aujourd'hui déconsidérées pour diverses raisons: dégradation qualitative des sols qu'elles occupent, qualité paysagère discutable, biodiversité presque nulle, grande fragilité face aux aléas susceptibles de ruiner les peuplements forestiers (et dont les incendies de la fin d'été 2007 en Grèce sont un exemple frappant). Les opérations de restauration mises en place dans maints massifs européens témoignent avec éloquence des revirements récents concernant différents aspects de la gestion forestière.

2 La restauration de ces forêts est d'abord menée dans une optique écologique : il s'agit de rétablir des écosystèmes en équilibre, en consolidant ou en réintroduisant dans les peuplements concernés une certaine diversité d'essences adaptées au milieu. Ces tentatives reflètent bien l'esprit des textes censés définir les bonnes pratiques en matière forestière, ainsi que les critères de certification appliqués au secteur bois, lesquels cherchent à concilier l'impératif économique au devoir écologique et social. En outre, une approche plus complète a parfois été tentée dans certains pays, et en 
particulier en Grande-Bretagne; il s'agit d'inclure des critères culturels dans la redéfinition des paysages forestiers.

3 Les paysages d'outre-Manche sont, il est vrai, peu boisés. Il y a moins d'un siècle, le taux de boisement de la Grande-Bretagne était réduit à $5 \%$ seulement; les plantations du XXe siècle n'ont remonté ce chiffre qu'à $12 \%$, avec d'importantes disparités régionales. C'est dire la rareté des Ancient Woodlands et la vénération qui leur est portée par les Britanniques... Peut-être est-ce pour cette raison que la restauration des forêts anglaises prend largement en compte, au-delà de l'économie et de l'écologie, la qualité paysagère et le patrimoine culturel lié à l'histoire forestière du pays.

4 L'exemple de trois forêts remarquables du point de vue patrimonial permet d'apprécier concrètement les idées britanniques sur ce qu'est le patrimoine forestier. La forêt de Hatfield, vaste massif périurbain situé au Nord de Londres, représente un conservatoire de pratiques anciennes où le retour à la nature n'est pas une option. La forêt de Glenmore est, à l'inverse, le type d'une forêt artificialisée que l'on souhaite ramener à un état d'équilibre relatif avec son environnement (restauration écologique), ou à un état supposé plus proche de la nature, selon l'expression consacrée. Enfin, dans la forêt de Sherwood, connue du monde entier, où se mêlent le mythe et l'histoire, s'expérimente le renouveau de certaines pratiques forestières traditionnelles : ici, la restauration écologique se double de considérations culturelles et patrimoniales.

\section{Les bonnes pratiques forestières : des critères nouveaux?}

\subsection{De la sylviculture à la foresterie : la gestion des forêts en quête de renouveau}

Les forêts européennes ont été fortement marquées par un mode d'exploitation des forêts essentiellement développé au XIXe siècle, mais dont les racines sont nettement plus anciennes. Ce modèle de gestion que l'on désigne parfois, et depuis peu, sous le nom de « classical forestry » ou "sylviculture classique » (ce qui suffit à montrer qu'il n'est plus vraiment au goût du jour) se base sur l'idée d'une forêt économiquement profitable, base d'un développement industriel gourmand en matières premières, qu'il s'agisse du bois lui-même ou des fibres ligneuses qui le composent. Cette idée s'est traduite, au XIXe puis au XXe siècle, par un mouvement d'ampleur européenne consistant à nier le rôle nourricier des forêts, jusque là étroitement associées aux terroirs cultivés dans le cadre d'une agriculture aux supports variés (Rochel 2006), et à rendre exclusive, ou presque exclusive la production de bois dans les objectifs assignés aux espaces boisés. Le mot «ligniculture » parfois utilisé est révélateur. En termes sylvicoles, le modèle classique se définit essentiellement par la priorité donnée aux peuplements aussi homogènes que possible, souvent monospécifiques, et généralement équiennes ou subéquiennes; ceci permet à la fois d'assurer une gestion facile, une récolte aisée, et une commercialisation rapide de lots homogènes pour l'industrie ou d'autres débouchés. Les essences privilégiées sont essentiellement les essences résineuses, qui se prêtent particulièrement bien à la fois aux objectifs et aux modalités techniques de la gestion mise en oeuvre (Johann, Rochel, et. al. 2004). 
Cette façon de mettre en valeur l'espace forestier relève d'une certaine idée utilitaire de la fonction des forêts. Cette vision dite classique est désormais battue en brèche par de nouvelles conceptions apparues dans les dernières décennies. Un intérêt plus grand porté à l'écologie, à la biodiversité, et à l'environnement en général, mène à chercher une définition d'une nouvelle gestion, parfois dite -sans grande imagination- "new forestry " et assimilée aux doctrines postmodernes ${ }^{1}$, plus souvent nommée foresterie durable. Le terme "foresterie ", longtemps inusité en France, s'est imposé récemment à la fois par contact avec l'anglais et du fait de la définition trop restreinte du mot "sylviculture », lequel n'englobe pas tous les aspects de la gestion et de l'exploitation des espaces boisés mais se limite à la conduite des peuplements. Le glissement de sylviculture (sens étroit) vers foresterie (sens large) pour désigner l'action des forestiers n'est pas neutre et montre justement l'entrée de la gestion des forêts dans une nouvelle complexité. Après avoir composé pendant longtemps la colonne vertébrale de l'action des forestiers, le binôme utilitaire conservation-production est désormais dépassé ou complété par d'autres idées et sensibilités. Selon Michael Williams (2000), l'assimilation de la nouvelle foresterie à la post-modernité se justifie par des similitudes de langage (obsession du complexe et de la diversité), mais aussi par l'impression de perte de repères : " like much post-modernist writing it is easier to say what the "new forestry" is not rather than what it is, and there is a strong sense of departure from old norms and explanations but little sense of destination ".

7 Il reste que la remise en cause de deux siècles au moins de gestion productiviste se traduit déjà sur le terrain dans le domaine de l'écologie forestière et de ses applications. Le respect de l'équilibre et de la complexité des écosystèmes est un fondement désormais assez généralement admis (Peterken 1996) et répond finalement à une préoccupation assez ancienne, comme l'atteste la vieille doctrine des sylviculteurs français de l'école de Nancy, «imiter la Nature, hâter son oeuvre ». La gestion durable est donc d'abord celle qui se refuse à artificialiser ou anthropiser la forêt de façon radicale, et qui au contraire cherche à respecter l'équilibre des écosystèmes diversifiés; il ne s'agit pas d'éviter toute empreinte humaine, mais de conserver ce que l'on appelle désormais une forêt subnaturelle.

8 Nulle part ce revirement ne s'observe mieux que dans les massifs où sont conduites des opérations dites de restauration, destinées à changer rapidement l'état des peuplements. Encore faut-il, compte tenu de l'enjeu, et de la forte inertie des écosystèmes forestiers, avoir une vision claire de ce qu'est cette foresterie durable. La préoccupation écologique ne suffit plus à définir les objectifs complexes et parfois confus de la nouvelle foresterie. Alors que les opérations dites de "restauration écologique » ont parfois conduit à ré-ensauvager (re-wild) les massifs, selon les mots de M. Hall (2000), ou du moins à leur rendre leur biodiversité, elles doivent aujourd'hui prendre en compte un éventail d'objectifs bien plus large. La définition de la foresterie durable, un temps confortablement limitée à des critères écologiques, se définit désormais par sa complexité, voire par une certaine confusion.

\subsection{A la recherche d'une définition complète de la foresterie durable}

9 La forêt intègre évidemment les domaines concernés par le développement durable. Le discours des forestiers revendique d'ailleurs un rôle pionnier en la matière: à lire certains d'entre eux, ce sont les forestiers qui auraient inventé le concept de durabilité, 
en recherchant dès les premiers balbutiements de la législation forestière la pérennité de la ressource forestière menacée par une exploitation minière. C'est oublier que l'idée de développement durable ne se suffit pas du caractère renouvelable des ressources et se définit généralement de façon plus complète. On évoque en particulier les désormais presque consensuels trois piliers du développement durable: l'écologique, l'économique, et le social. Sans vouloir nier la filiation entre recherche forestière, législation forestière et gestion durable des milieux (Galochet, Rochel 2008), les visions contemporaines de la gestion durable des forêts ne se contentent donc pas d'évoquer la pérennité productive de la forêt en tant que ressource; elles n'y ajoutent pas que des considérations environnementales; elles incluent un aspect social, qui dépasse le seul confort de vie, pour inclure la justice sociale mais aussi, plus récemment, le respect des cultures. Cette dernière idée a émergé lentement: la culture semblait mal s'intégrer dans le concept de durabilité, si ce n'est qu'une certaine gestion des forêts ne sera acceptée, et légitimée localement que si elle respecte un certain nombre de conditions, comme le respect de pratiques traditionnelles non destructrices ou des croyances ancrées dans l'espace forestier. Derrière le concept de durabilité se profile celui de gouvernance ; deux lieux communs, peut-être, et pourtant hautement légitimes.

10 La recherche (inachevée) de critères de définition d'une foresterie durable s'est faite par étapes, sur plus de quinze ans désormais. Les premières réflexions à l'échelle continentale ont conduit, peu après le Sommet de la Terre, à la définition de 6 grands principes dénommés guidelines for the sustainable management of forests in Europe, sous l'égide de la $\mathrm{MCPFE}^{2}$ et proclamés, sans produire beaucoup d'échos au delà des sphères forestières, à Helsinki en 1993 ("Déclaration d'Helsinki »). La déclaration dite de Lisbonne, cinq ans plus tard, aboutissait à un texte qui semblait plus élaboré et qui, surtout, accordait davantage d'importance aux aspects sociaux et culturels, dans la droite ligne de l'évolution du concept de développement durable lui-même. Enfin, en 2003, à la suite de sa quatrième réunion à Vienne, la MCPFE contribuait encore à renforcer la place des paramètres sociaux et culturels parmi les critères de bonne gestion forestière (Déclaration de Vienne, 2003). Ce débat n'est évidemment pas qu'européen et reflète la réflexion menée à l'échelle mondiale, en particulier au sein de la F.A.O.

11 Quels que soient les critères retenus, le concept de gestion durable forestière (Sustainable Forest Management, SFM) dépasse donc les seuls domaines de l'écologie et de l'économie, pour prendre en compte les sociétés et leurs cultures. Ce dernier thème a peut-être été sous-considéré dans les premières réflexions consacrées à la forêt durable. Les travaux de recherche se sont multipliés dans ce sens et l'histoire de l'environnement, alléchée par une thématique culturelle devenue très porteuse, n'a pas été en reste : séminaire «forestry and our cultural heritage » de la MCPFE à Sunne, 2005 ; colloque « cultural heritage and sustainable forest management » à Florence, 2006 ; colloque "woodland cultures in time and space», à Thessalonique en 2007. Des publications nombreuses dans les dernières années témoignent de la vitalité d'un domaine de recherches qui doit beaucoup aux évolutions politiques récentes (Watkins 1998; Agnoletti 2000; Agnoletti 2006). On sait que même une forêt comme Białowieża (Pologne), trop souvent citée comme une quasi-» forêt vierge », est fortement marquée par l'activité humaine. Des massifs emblématiques de la sylviculture française comme les forêts de Haye et Tronçais, masquent les traces fossiles de terroirs largement cultivés pendant plusieurs siècles, et la définition traditionnelle des "vieilles forêts " 
ou «ancient woodlands » par opposition aux reboisements récents apparaît troublée par les découvertes récentes (Dupouey et. al. 2007). La naturalité des écosystèmes boisés pose question; l'espace forestier relèverait-il essentiellement d'une construction historique, et donc du patrimoine culturel ?

Cette prise de conscience n'est pas encore généralisée. En 2004, l'IFN mettait à jour son cahier portant sur « les indicateurs de gestion durable des forêts françaises ». On aurait pu espérer y trouver un jugement sain sur la gestion pratiquée dans les forêts françaises. Or, la publication reste très statistique, très quantitative : était-il bon de confier la réalisation d'un tel document à l'IFN seul ? Le plan reprend tels quels les six critères d'Helsinki; seul le critère 6.11 semblait devoir se rapporter à la question culturelle; on ne lit rien d'importance dans le très court paragraphe qui s'y rapporte, rien par exemple sur la qualité des paysages ou les vestiges archéologiques... alors qu'on y disserte par exemple sur les arboretums, dont la valeur culturelle reste à démontrer!

13 Les six critères de durabilité de la gestion forestière avancés à Helsinki en 1993, trop souvent encore cités comme une référence actuelle en matière de bonne gestion forestière, sont donc dépassés ; encore faut-il que l'aspect culturel de la «durabilité » intègre, au delà du discours, les pratiques sur le terrain. Or, si les réflexions ont considérablement avancé, la pratique n'en est qu'à ses balbutiements, que ce soit dans la gestion ordinaire ou les opérations de restauration. C'est peut-être pour cette raison que les critères de certification forestière n'abordent pas encore la question, si ce n'est de façon très secondaire. Pourtant, des modèles de gestion respectant l'histoire, les héritages paysagers, les techniques, pratiques et croyances locales et plus généralement la culture forestière relative aux sites concernés peuvent être trouvés ici et là et, pour diverses raisons, la Grande-Bretagne se révèle en pointe sur le sujet.

\section{Le patrimoine forestier en Grande-Bretagne : un enjeu particulier}

\subsection{Un patrimoine paysager façonné par les pratiques traditionnelles}

14 Si l'enjeu patrimonial représenté par les forêts britanniques et en particulier les ancient woodlands ${ }^{3}$ semble particulièrement important, c'est d'abord parce qu'au cours des siècles, s'est forgé un modèle de gestion qui se distingue assez nettement des pratiques les plus communes sur le continent. En effet, le modèle britannique traditionnel du massif «forestier» juxtapose deux types de paysages nés de pratiques complémentaires : les taillis et les parcs ou wood-pastures.

15 Les taillis (coppices) sont des parcelles boisées, traitées en taillis ou en taillis sous futaie, et principalement gérées de façon à produire du bois. Le produit essentiel est bien sûr, compte tenu du traitement utilisé, le bois de feu à destination industrielle ou domestique. Habituellement, un compartiment de taillis est exploité chaque année, et la durée du cycle sylvicole (révolution du taillis) est donc égale au nombre de parcelles ainsi gérées. Mais le taillis est aussi un lieu de chasse, traversé par les allées (rides) qui facilitent les activités cynégétiques, parfois sous la forme d'un réseau en étoiles de chasse. 

surface est occupé par une végétation herbacée, ce qui ne leur ôte pas pour autant leur vocation forestière. En effet, cette strate basse pâturée par les troupeaux domestiques, semi-domestiques (daims) ou sauvages est surmontée d'une seconde strate, plus ou moins dense selon les cas, d'arbres régulièrement émondés ou, surtout, étêtés (pollards, figure 1). Ainsi peut-on associer, sur une même surface, un cheptel domestique important, une réserve de gibier non moins importante, et une production de bois non négligeable. Les wood-pastures peuvent représenter la plus grande partie d'un massif forestier: Ashdown Forest, par exemple, occupe dans le Sussex un total de 2500 hectares, dont 1365 sont occupés par une végétation basse de type lande plus ou moins piquetée d'arbres épars en 2005 (source : Ashdown Forest Strategic Forest Plan 2008-2016, p. 5).

17 Ces deux modèles ne sont pas toujours bien délimités, et s'interpénètrent souvent; les pollards sont aussi présents dans les taillis, et les animaux peuvent généralement passer des espaces ouverts aux compartiments boisés, qui ne sont clos que pendant quelques années après l'exploitation du taillis, afin de protéger les rejets de l'abroutissement. L'ensemble coppices + woodpastures forme un objet patrimonial diversifié et avenant, très cher au cœur des Britanniques.

Figure 1. Une pratique forestière patrimoniale : l'étêtage (pollarding).

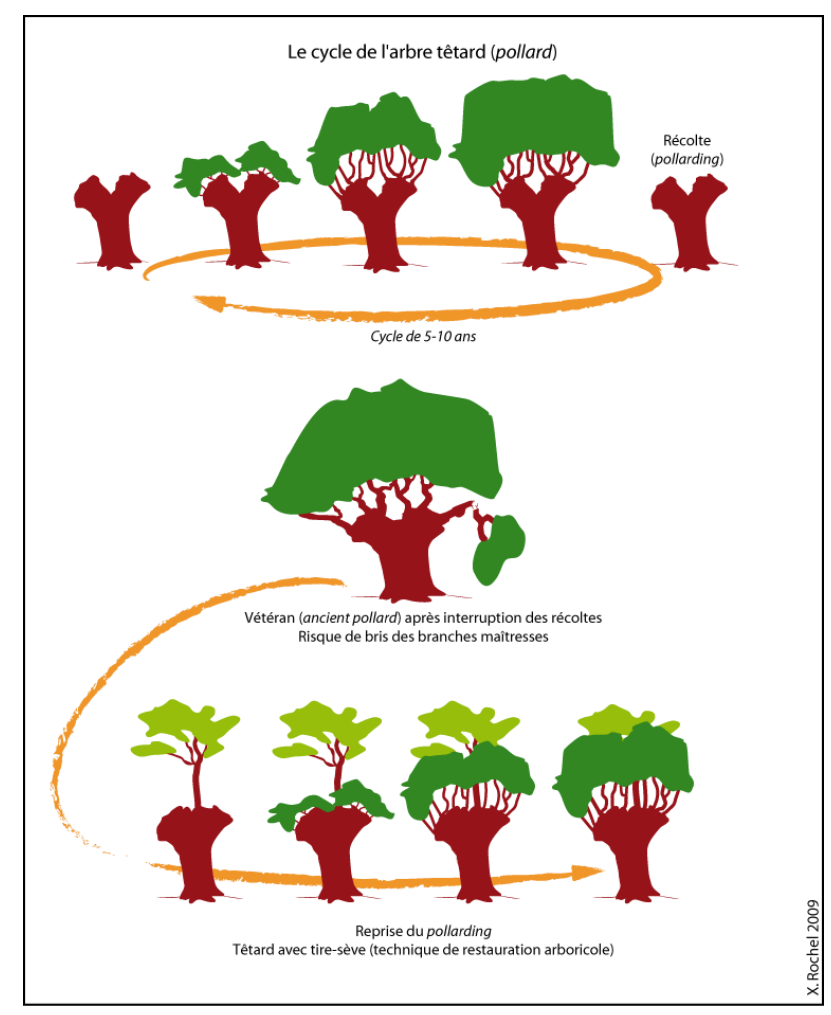

\subsection{Les ancient woodlands, un patrimoine choyé}

18 Ce patrimoine forestier marqué par l'histoire est d'autant plus choyé qu'il est rare. A l'instar du reste de l'Europe, la Grande-Bretagne a connu au cours de son histoire médiévale et moderne un recul progressif de son couvert forestier, jusqu'à atteindre 
un minimum au XIXe siècle. Mais ce minimum fut ici particulièrement marqué. La forêt occupait encore 15 à 20\% de l'Angleterre du Domesday book (XIe siècle; Darby 1977), mais $10 \%$ seulement dès 1350 . Le taux de boisement de l'Angleterre, du Pays de Galles et de l'Ecosse réunis était de $5 \%$ seulement en 1900 (Smout 2003, Williams 2006)... Le manque de bois, matériau stratégique s'il en est au temps de la marine britannique triomphante, ne manqua pas d'inquiéter les observateurs dès le XVIIe siècle. "No wood, no kingdome " disait Arthur Standish en 1611; une citation qui peut être rapprochée du fameux (et peut-être imaginaire) "la France périra faute de bois » attribué à Colbert. En 1669, poussé par l'inquiétude générale, John Evelyn publiait son Sylva, or a Discourse on Forest-Trees, que les anglo-saxons considèrent parfois comme le premier véritable traité de foresterie.

Les bois étant alors rares... et chers, on considère qu'ils apportent de la beauté au paysage ; espaces cynégétiques, propriétés aristocratiques par excellence, ils aident à l'affirmation sociale des élites. Désormais, la forêt est un décor et un luxe autant qu'une ressource économique. Les besoins en bois du royaume sont satisfaits grâce aux importations. Les bois prussiens, polonais, scandinaves, arrivent en masse dans les ports de l'Est anglais. Ce « baltic trade » prend une importance telle que la dénomination anglaise de l'épicéa, spruce, viendrait du mot Prussia et serait ainsi un témoignage de ce qui fut l'une des filières privilégiées pour l'importation des bois de construction et des bois de mâture. Le Nouveau Monde, lui aussi, apporte malgré la distance des ressources en bois de qualité désormais difficiles à obtenir sur le Vieux Continent ; mais dès 1775, le bois est aussi cher en Nouvelle-Angleterre qu'en métropole (Williams 2006 p. 212)! A cette date, l'usage croissant du charbon de terre permet heureusement déjà de répondre aux besoins de l'industrie, et contribue ainsi à réduire le rôle producteur des forêts.

Il y a donc peu de forêts, en Grande-Bretagne, dont le couvert boisé ait été continu dans les derniers siècles. Ces "ancient woodlands", dont l'état boisé est attesté depuis au moins l'an 1600 selon la définition classique outre-Manche, sont si peu nombreux qu'on a pu tenter de les inventorier de façon exhaustive dans les années 1980 (AWI, Ancient Woodlands Inventory), ce qui eut été évidemment impensable sur le Continent, du moins à l'échelle d'un Etat entier.

21 Les évolutions du XXe siècle, bien que quantitativement favorables pour l'espace boisé, contribuèrent encore à renforcer la rareté, et donc la valeur culturelle des paysages forestiers préservés, par le biais des conversions en futaies résineuses. L'intérêt du bois de feu étant très limité, l'élevage en milieu forestier (boisé ou semi-boisé) se révélant peu intéressant, les pratiques continentales d'enrésinement trouvèrent en GrandeBretagne un terrain d'action privilégié. Au total, $40 \%$ des coppices de Grande-Bretagne firent l'objet d'un enrésinement en plein, généralement en épicéas (Picea), en douglas (Pseudotsuga), ou en pins (Pinus). Désormais, l'arbre le mieux représenté en GrandeBretagne est un résineux originaire de la côte ouest américaine, l'épicéa de Sitka (Picea sitchensis)...

On distingue donc parmi les ancient woodlands deux catégories: d'une part, les native woodlands, dans lesquels règnent encore les essences autochtones (native species) telles que le chêne (Quercus sp.), le charme (Carpinus betulus), ou l'orme (Ulmus sp.); d'autre part, les planted ancient woodlands (P.A.W.), qui ont été enrésinés. A en croire English Nature, sur les 340000 ha considérés comme des ancient woodlands en Angleterre, seuls 200000 ha sont peuplés d'espèces autochtones et entrent ainsi dans la catégorie des 
forêts dites semi-naturelles, tandis que le reste est occupé par des plantations très majoritairement résineuses. Ceci explique la vénération apportée aux native woodlands, et l'importance particulière de la problématique de la restauration écologique dans les P.A.W. (Smithers 2002). Dès 1985, le principe d'une politique en faveur des essences feuillues, qu'on désigne aujourd'hui sous le nom de broadleaves policy, était proclamé à l'échelle nationale.

Les transformations des forêts britanniques au cours du XXe siècle contribuent aussi à expliquer l'amour immodéré porté aux vieux arbres, forestiers ou non, qui représentent une sorte d'âge d'or de paysages bouleversés. Parmi ces vénérables ancêtres, deux l'emportent sur les autres en renommée: le Glastonbury Thorn (Crataegus monogyna), et le Major Oak de Sherwood (Quercus robur, photo 1). Si l'on écarte le Glastonbury Thorn qui ne peut être qualifié d'arbre forestier, le vétéran le plus significatif est le Major Oak, sur le domaine de Thoresby identifié comme un vestige de l'ancienne forêt de Sherwood: il s'agirait, dit la légende, du chêne sous lequel Robin des bois et ses compagnons avaient coutume de se retrouver. Mais ce sont des milliers d'ancient trees vieux de plusieurs siècles sur lesquels se porte l'intérêt des amoureux des arbres.

Photo 1. Le Major Oak (Cliché Xavier Rochel, 2005).

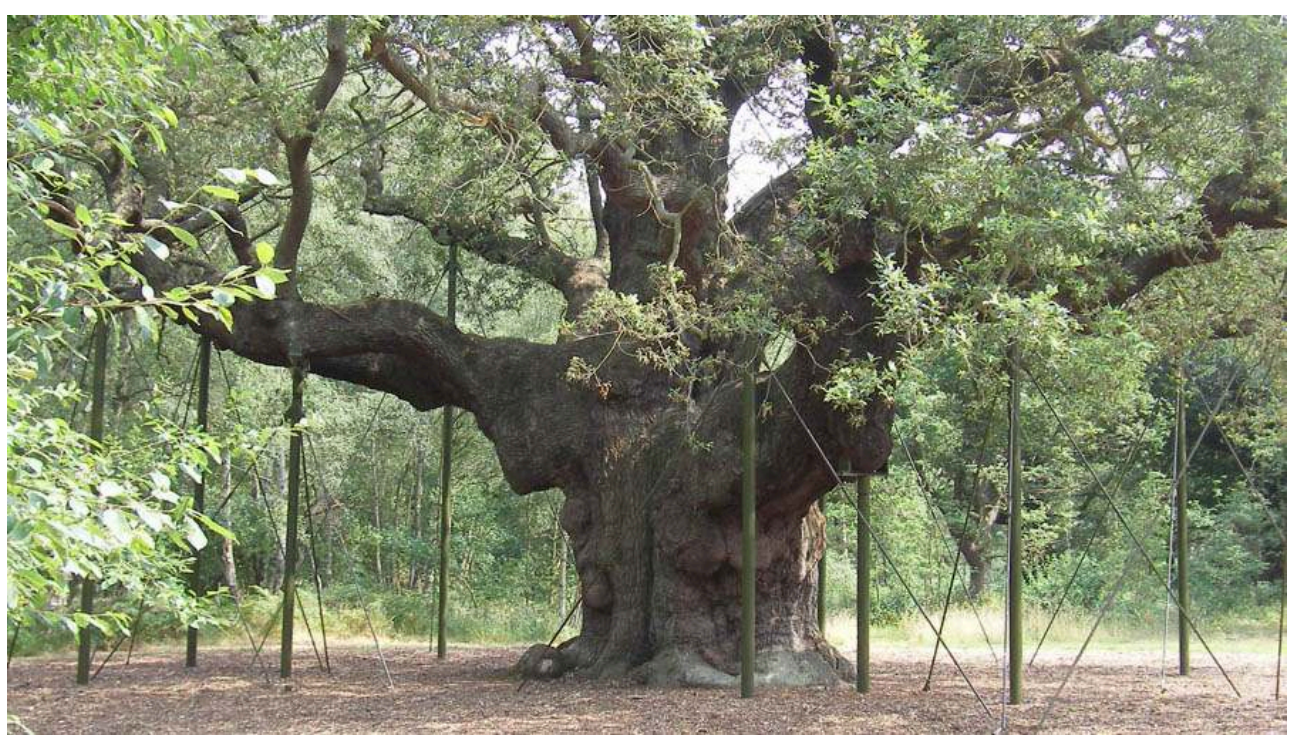

Ces vénérables vétérans ont très souvent le même visage. Il s'agit généralement d'anciens pollards, chênes, frênes, ou autres essences feuillues, avec un tronc difforme (bole ou bolling) de 3 à $6 \mathrm{~m}$ de haut dont la « tête » boursouflée est héritée des anciennes pratiques d'étêtage. Celles-ci ayant cessé depuis des décennies, les branches ont grossi sans entrave jusqu'à approcher du point de rupture: très souvent, les branches maîtresses cassent sous leur propre poids. Tout aussi souvent, les arbres pénalisés par leur port trapu sont surcimés et étouffés par leurs voisins, qui poussent sans contrainte vers la lumière. Ceci se produit surtout lorsque les pollards sont noyés dans les plantations résineuses, ce qui arrive souvent: en général, les enrésinements ont épargné les plus gros vétérans (le coût du bûcheronnage eût été prohibitif!) mais les ont condamnés à un lent déclin.

Bien entendu, ont été expérimentées des techniques de restauration arboricole, inaugurées à la fin du XXe siècle, par exemple à Ashton Court, y compris par la reprise 
de l'étêtage (restoration pollarding). Faut-il vraiment, pour retrouver les paysages d'antan, toucher à ces vieux arbres et leur faire subir à nouveau les pratiques qui leur ont donné leur forme? L'idée d'une reprise du pollarding, et d'une taille sévère sur ces silhouettes pourtant pittoresques découle directement de l'approche patrimoniale des ancient trees, dont la valeur biologique (comme habitat pour l'entomofaune par exemple) ou esthétique n'est pas méconnue, mais dont l'intérêt patrimonial réside d'abord dans l'importance historique de l'étêtage en Grande-Bretagne : les pollards d'il y a deux siècles n'étaient pas ces vétérans difformes, mais des arbres en pleine santé, aux branches menues, dont la capacité productive était pleinement exploitée. De même, les bois de l'âge médiéval et moderne n'étaient pas des lieux sauvages et impénétrables, mais des espaces largement ouverts, périodiquement taillés à ras du sol, où s'élevaient à de larges intervalles des arbres réservés (standards) et des pollards de tous âges. Ici s'affrontent donc deux visions de la forêt, l'une centrée sur l'histoire et le patrimoine, l'autre orientée vers un imaginaire tout à fait différent où l'empreinte de l'homme reste discrète au milieu de vieux arbres tordus et de frondaisons presque inviolées.

\subsection{Une histoire partiellement masquée par l'imaginaire}

26 La perception du paysage forestier en Grande-Bretagne ne se résume donc évidemment pas à une histoire très matérielle et aux vestiges qui en sont hérités. La réalité observable, ou reconstituée par l'histoire des forêts, est pour partie masquée ou complétée par une culture de la forêt propre à la Grande-Bretagne, bâtie au cours des siècles et confortée par une riche littérature. Cet imaginaire est évidemment marqué par l'idée du bois sauvage et hostile à l'humanité, mais bien moins qu'en Europe continentale ; après tout, le loup fut exterminé en Grande-Bretagne dès la fin du XIVe siècle. Davantage qu'un espace hostile, la forêt est d'abord un refuge pour les opprimés et un symbole de liberté.

27 Simon Schama (1995) a minutieusement analysé la constitution progressive de cet imaginaire centré autour de ce qu'il appelle le greenwood, ou ce que Richard Muir appelle, à l'ancienne, le Merrie Greenwoode défini comme « un lieu de joyeuse dérobade loin des abus féodaux " (" a place of joyful escape from the impositions of feudalism », Muir 2005). Robin Hood, ce «Robin la Capuche » inexactement mais opportunément traduit en "Robin des Bois ", présent dans la littérature dès le XIVe siècle, est le personnage emblématique de ce refuge boisé. Mais plus que le trop romanesque Robin, c'est le personnage de Henry Hastings qui incarne sans doute le mieux l'esprit du greenwood. Ce nobliau, forestier en charge d'un secteur de la New Forest, rougeaud et mal habillé, sans cesse accompagné de ses chiens et chats, truculent et libertin jusqu'à l'outrance, chassa le cerf à courre jusqu'à quatre-vingt ans passé, et vécut centenaire (Schama 1995). A travers la communauté créée par Robin des Bois, ou autour de la table pittoresque de Henry Hastings, le greenwood représente une forme de sociabilité invincible et joyeuse qui se crée contre l'intrus; il symbolise la liberté des bois et des landes, héritée des temps pré-normands, incarnée par les chasseurs (ou les braconniers) et la communauté solidaire des gens des bois, à l'encontre du vice et de la corruption d'une noblesse cruelle, des agents royaux corrompus, et d'une société urbaine intruse et maléfique.

28 Le fils de Guillaume le Conquérant, Guillaume le Roux, en fit les frais. Personnage avide de chasse, méprisant pour les habitants des bois, il incarne la naissance d'un droit cruel censé protéger le gibier royal contre le braconnage - ou plutôt destiné à faire de la 
chasse une prérogative princière dans les immenses territoires nouvellement érigés en forêts royales. Il ne pouvait contrarier ainsi les libertés du greenwood sans subir l'intervention réparatrice de la Providence. Au cours d'une chasse, une flèche destinée à un chevreuil ricocha sur un chêne (phénomène hautement improbable et donc forcément surnaturel) pour aller se planter dans le corps du roi, qui en mourut (Rackham 1990, Schama 1995). Cet épisode mythique représente le fonds ancien d'un imaginaire ultérieurement développé et complété par la littérature romanesque : on pense évidemment à Walter Scott (Ivanhoe), mais aussi à Robert Louis Stevenson (The Black Arrow) par exemple.

Ainsi la redéfinition de la foresterie en Grande-Bretagne doit-elle prendre en compte un imaginaire riche et prégnant, en plus d'une conscience patrimoniale exacerbée et des aspirations à l'équilibre biologique. Cette triple préoccupation ne va pas sans susciter des interrogations et des conflits. Ainsi la restauration en cours des paysages de la forêt d'Ashdown a-t-elle déjà montré combien la perception populaire d'un certain idéal forestier pouvait contrarier une gestion centrée sur l'idée de patrimoine culturel ou historique. Il s'agissait, en l'occurrence, de faire face énergiquement à l'enfrichement et à la progression de l'arbre dans les espaces ouverts autrefois gérés selon le modèle du parc ou des wood-pastures. Les tentatives de défrichement, peut-être insuffisamment expliquées auprès du public, ont conduit à d'énergiques manifestations, au cours desquelles les dégagements ont été comparés à l'Holocauste ou à la bataille de la Somme. Ceci montre à quel point le modèle historique, relativement peu boisé, de la gestion forestière ne correspond plus à ce que l'on considère comme une «vraie forêt » dans une partie de la société anglaise ${ }^{5}$. Des conflits similaires ont d'ailleurs eu lieu dans la New Forest et le Surrey.

\section{Trois massifs modèles pour une gestion forestière en renouveau}

Les trois exemples qui suivent aident à bien cerner, sans aller trop loin dans les détails monographiques, l'esprit dans lequel sont menées les opérations de restauration dans les forêts britanniques; ils s'inscrivent à la fois dans le cadre européen de la redéfinition de la gestion forestière, et dans le contexte très particulier propre aux îles britanniques.

\subsection{Un conservatoire : la forêt de Hatfield}

La forêt de Hatfield présente la particularité d'avoir été peu affectée par les pratiques de la ligniculture. Les plantations en essences résineuses n'ont touché qu'une part infime des peuplements, tandis que les pratiques traditionnelles y ont été menées assez longtemps pour pouvoir être reprises de nos jours sans grandes difficultés, presque comme si aucune interruption n'avait eu lieu. Cette évolution, ou plutôt cette inertie si rare dans les forêts d'outre-Manche s'explique en particulier par l'acquisition du massif par une organisation à but non lucratif, le National Trust for Places of Historic Interest or Natural Beauty (généralement connu sous le nom abrégé de National Trust), dès 1924. Dans ce sens, Hatfield est un conservatoire particulièrement intéressant en ce qu'il présente, sur un peu plus de 400 hectares, le visage qui était celui des forêts anglaises jusqu'aux bouleversements du XXe siècle. 
Le massif, longuement étudié par Rackham (1989), présente donc les deux faciès évoqués plus haut. La partie véritablement boisée était autrefois divisée en 16 parcelles de taillis, ou coppices, dont une était exploitée chaque année, à tour de rôle. Les défrichements ultérieurs ont réduit le nombre de ces parcelles à 12. Le reste du massif est composé par ce que Rackham appelle des plains, c'est-à-dire des pâturages qui s'étendent en une forme digitée d'un bout à l'autre du massif (photo 2). Selon le modèle des wood-pastures, ces pâturages sont piquetés d'arbres nombreux, généralement des pollards de diverses essences feuillues, parfois très vieux et présentant des formes spectaculaires.

Photo 2. Les pâturages à arbres têtards de la forêt de Hatfield (cliché X. Rochel, 2006).

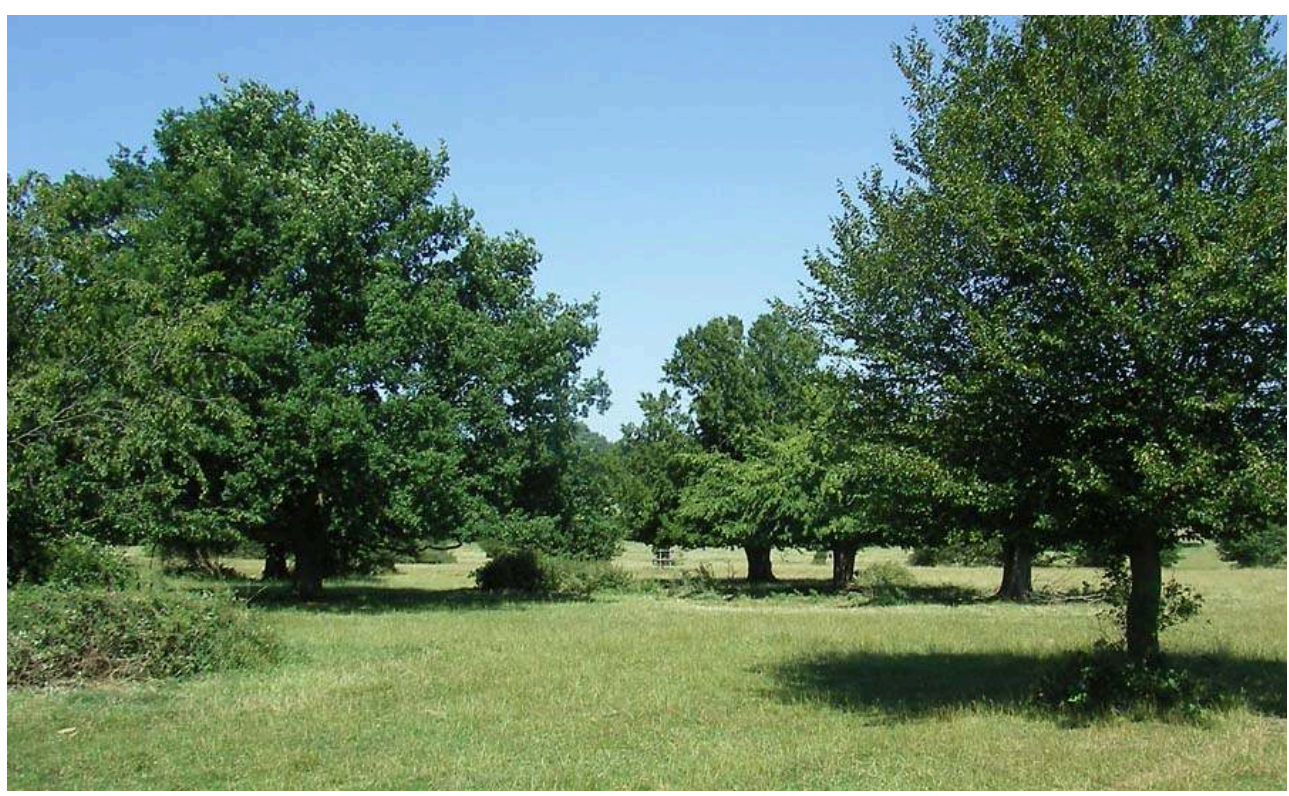

La très bonne conservation du massif en fait une attraction touristique très courue, l'un des sites phares du National Trust, d'autant que le massif se trouve à proximité immédiate de l'agglomération londonienne. Un parking payant assure le financement du maintien des pratiques traditionnelles et la gestion de la fréquentation. De fait, la gestion se base sur le principe de la continuité et la restauration proprement dite du massif se limite à peu de choses. Il s'agit essentiellement d'achever la résorption des faibles surfaces de plantations résineuses (figure 2), dont il reste quelques traces dans les taillis de Spittlemore, Collin, et Emblem; les plantations de Table Coppice ont déjà été totalement converties en essences feuillues autochtones. Il s'agit également de maitriser, voire d'inverser la tendance à la progression des broussailles et de la friche boisée aux dépens des espaces ouverts, ce qui impose une gestion dynamique du pâturage sur la surface du massif. 
Figure 2. La forêt de Hatfield, un conservatoire paysager (X. Rochel, sources : Rackham 1989, Ordnance Survey, images NAVTEQ, observations personnelles 2006)

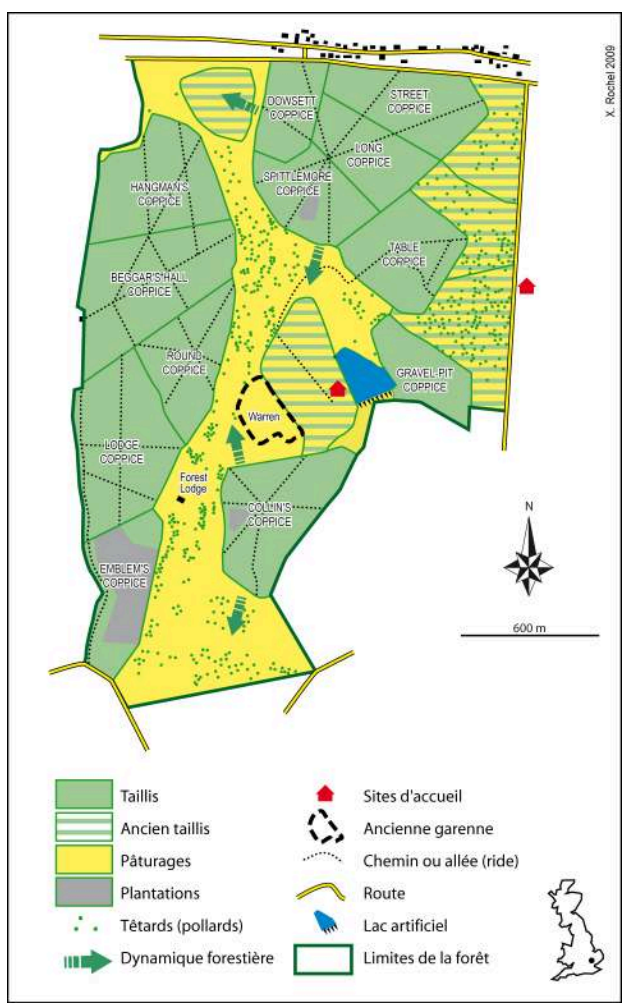

\subsection{Une restauration écologique : la forêt de Glenmore}

Contrairement à Hatfield, le massif de Glenmore, dans les Highlands d'Ecosse, est représentatif de ces massifs forestiers en partie plantés, très marqués par la ligniculture du XXe siècle, qui connaissent aujourd'hui d'importants changements dans leur gestion en raison des évolutions du contexte économique et surtout culturel mentionnées précédemment. Il s'agit d'un domaine de 3566 hectares dont 1345 boisés, sur le flanc Nord des Cairngorms, le second massif montagneux le plus élevé d'Ecosse après le Ben Nevis. Le domaine présente deux caractéristiques qui en font un site clé parmi les forêts écossaises: d'une part, il renferme une partie notable des dernières forêts subnaturelles de Pin sylvestre (Caledonian pinewoods, peuplées de Pinus sylvestris); d'autre part, il est au cœur d'un complexe touristique de premier plan avec réseau de chemins de randonnée, activités de loisirs lacustres et station de sports d'hiver. Cette délicate association en un même site d'une grande richesse écologique et d'une fréquentation importante explique que la forêt de Glenmore fut le deuxième massif érigé en Forest Park en Grande-Bretagne. La fréquentation était estimée à 10000 visiteurs par an il y a quelques décennies; elle s'élèverait aujourd'hui à 350000 visiteurs par an.

Le massif a hérité une grande partie de ses paysages actuels d'une histoire essentiellement marquée par l'importance actuelle et passée du pâturage, qui a fortement transformé les peuplements, et par les plantations monospécifiques en essences locales ou importées. Les paysages sont donc fortement anthropisés, et apparaissent de prime abord très marqués par un modèle de gestion orienté vers la ligniculture. Ce visage, d'autant plus inacceptable que la fréquentation est forte et la 
pression du public considérable, a évidemment imposé un changement de gestion dès les années 1990, qui fut officialisé et accéléré par l'établissement d'un plan de gestion orienté vers la restauration du massif en 2005. Le projet mis en œuvre est organisé autour du concept de multifonctionnalité. La fonction de production du massif n'est pas véritablement remise en cause, mais la gestion se veut plus respectueuse de l'environnement : le projet répond au modèle de la restauration écologique.

Le problème de la "forêt calédonienne ", cette forêt de pins sylvestres (Caledonian pines) qui est la forêt écossaise par excellence, occupe une place centrale dans la gestion nouvellement mise en place. Il s'agit désormais d'éliminer, de façon progressive comme il se doit dans le contexte forestier, les essences allochtones, parmi lesquelles l'épicéa de Sitka occupe la première place. Cette transformation se fait et a déjà commencé à se faire par coupes rases, suivies d'une replantation lorsque la forêt ne peut se régénérer par elle-même grâce aux arbres voisins ou aux banques de graines du sol. Il est même prévu d'opérer des arrachages manuels d'arbustes indésirables. A dire vrai, cette politique n'est pas vraiment nouvelle : c'est dès 1991 que des opérations ayant pour objet d'éliminer les essences introduites lors des principales phases de plantation (1924-1933, 1950-1967, et 1985-1990) furent entreprises. Le pin sylvestre domine déjà 967 hectares, soit $71 \%$ de la surface boisée actuelle. Ces surfaces seront donc étendues, et une gestion "à faible impact " y sera pratiquée, en favorisant les pins sylvestres locaux aux dépens des pins plantés qui sont jugés génétiquement moins intéressants. En outre, afin de créer un massif cohérent de surface importante, les liens avec les pineraies voisines de Rothiermurchus et Abernethy doivent être reconsidérés, notamment à travers la constitution d'un corridor écologique vers Abernethy (figure 3).

La restauration écologique ne s'arrête évidemment pas à cet objectif prioritaire. Les essences feuillues doivent également être favorisées. Les espaces ouverts doivent être maintenus par une maitrise de la dynamique du pin vers les zones d'altitude. Une attention particulière est portée à la biodiversité animale : il s'agit de recréer une forêt peu accueillante pour l'écureuil gris américain, qui menace l'existence des écureuils roux locaux par exemple. A l'inverse, les peuplements doivent être aussi accueillants que possible pour le Tétras (Capercaillie, Tetrao urogallus).

La forte fréquentation complique bien évidemment la gestion envisagée. Il s'agit de limiter les usages récréatifs (donc la fréquentation) dans les espaces de plus haute valeur écologique, comme les native pinewoods, pour les guider, à l'inverse, vers les espaces plus largement anthropisés au cours du XXe siècle. Le dossier n'aborde pas la difficulté que vont représenter, à cet égard, les lourds travaux de restauration entrepris dans cette zone dont les paysages risquent d'être peu avenants dans les quelques décennies à venir, tandis que les boisements calédoniens préservés pourront naturellement attirer davantage le randonneur.

Pourtant, le dossier comporte un volet paysager important. La forme des coupes pratiquées ne doit pas choquer, mais doit s'intégrer dans le paysage, en présentant le moins possible de formes géométriques: "felling proposals have been designed to give interlocking shapes that broadly reflect the landform » (Forest Design Plan, draft 2005). En outre, l'ensemble du projet a fait l'objet d'une large concertation à travers un appel au public. Le dossier publié comportait, en particulier, des simulations informatiques en trois dimensions de l'évolution du paysage dans les prochaines décennies, jusqu'à 2030. On constate d'ailleurs, sur les images satellitales récentes, que les opérations forestières se sont jusqu'ici concentrées non dans les parcelles occupées par les résineux 
exotiques, mais dans celles les plus visibles ou les plus proches des points les plus fréquentés (figure 4). On est donc déjà au-delà de la classique restauration écologique, laquelle est traditionnellement la chasse gardée d'un milieu d'experts particulièrement réduit.

Figure 3 : le massif de Glenmore (Source : Glenmore Forest Design Plan - draft 2005, Ordnance Survey, images NAVTEQ, observations personnelles 2005)

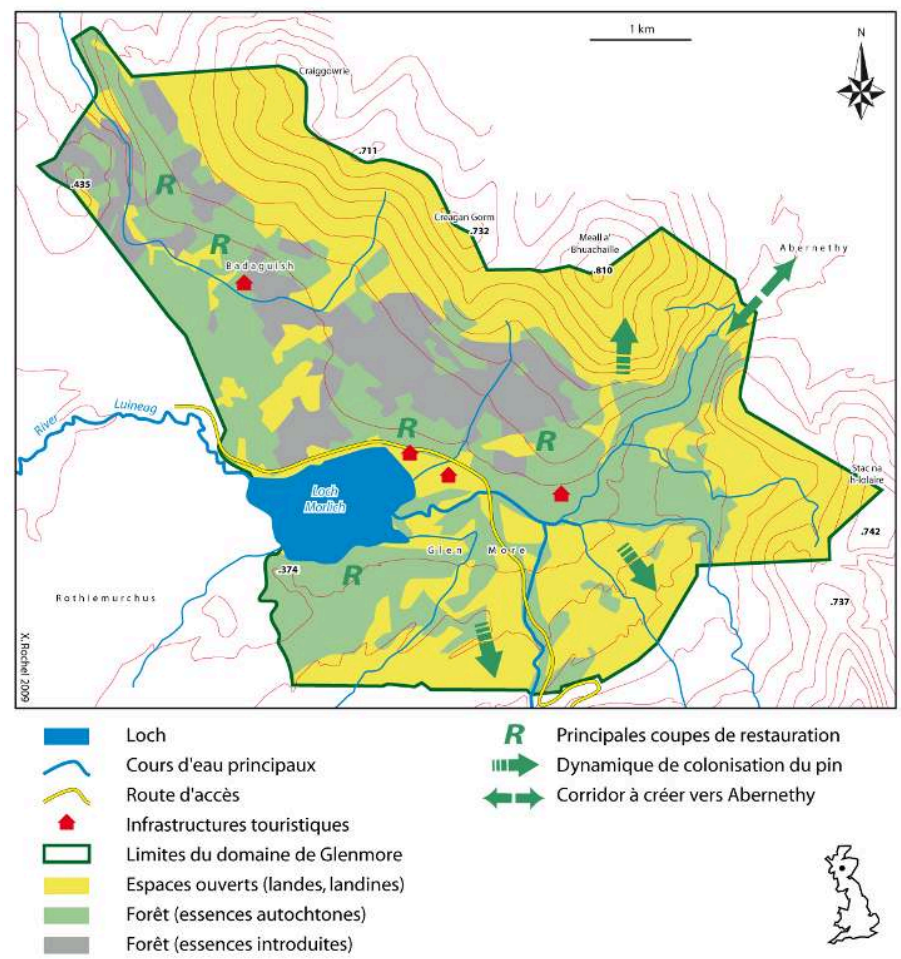

\subsection{Une restauration pilote en matière culturelle : la forêt de Sherwood}

La forêt de Sherwood, dans le Nottinghamshire, est évidemment d'abord une forêt mythique; mais le mythe de Robin et de ses compagnons s'inscrit, comme souvent, dans une réalité historique et géographique indéniable. Le nom de Sherwood est connu pour la première fois dans un texte de 958 sous la forme Sciryuda qui a le même sens que Sherwood : forêt appartenant au shire (comté). Le massif ainsi désigné devint, après 1066, une forêt royale, l'une des forêts préférées des rois anglais, incluant non seulement un important massif boisé, mais également des wood-pastures, des cultures, des prés, des villages, et trois parcs à gibier fermés. Bien qu'en grande partie défrichée, la forêt resta propriété royale jusqu'au XIXe siècle où elle fut intégrée à un domaine privé (estate) ${ }^{6}$. Les dernières chasses royales eurent lieu dans le courant du XVIIe siècle. A l'inverse de la forêt de Hatfield, et à l'instar de la grande majorité des forêts anglaises, elle ne conserva pas les pratiques et les paysages traditionnels, malgré l'attraction touristique qui débuta dès le XIXe siècle. La forêt, intégrée à un domaine privé d'assez faible taille, dut s'adapter aux besoins financiers de ses propriétaires et fut ainsi fortement enrésinée tout au long du XXe siècle. En outre, la pression industrielle locale favorisa la transformation des peuplements à travers la demande en 
bois de mine résineux: le massif jouxte une importante exploitation de charbon, la Thoresby Colliery, en partie implantée sur des surfaces autrefois boisées. Les intendants successifs en charge de la gestion forestière implantèrent donc très largement le pin sylvestre et le mélèze, dans un premier temps, puis, après-guerre, le pin laricio qui marque encore très fortement les paysages actuels (Kottler, Watkins, Lavers 2005).

Dès 1969, un Forest Park était créé sur le domaine. Mais le tournant dans la gestion se situe vers 1980, avec la montée inexorable de l'intérêt porté à la conservation des paysages et des écosystèmes. En outre, les aides publiques, particulièrement importantes dans l'évolution de la gestion, ne portent plus sur les plantations et la ligniculture, mais plutôt sur les actions en faveur de la conservation du patrimoine, qu'il soit naturel et culturel. La forêt étant unanimement jugée exceptionnelle pour son rôle historique, pour la légende de Robin, mais aussi pour ses caractères écologiques, ne pouvait évidemment pas être gérée comme n'importe quelle forêt anglaise. $Y$ ont été mis en place une réserve naturelle, et d'importantes infrastructures d'accueil, co-gérées par les propriétaires (privés ou publics) et les organismes publics de gestion forestière qui se partagent les rôles occupés en France par l'ONF.

Photo 3. L'entrée de la forêt de Sherwood (cliché Xavier Rochel, 2006).

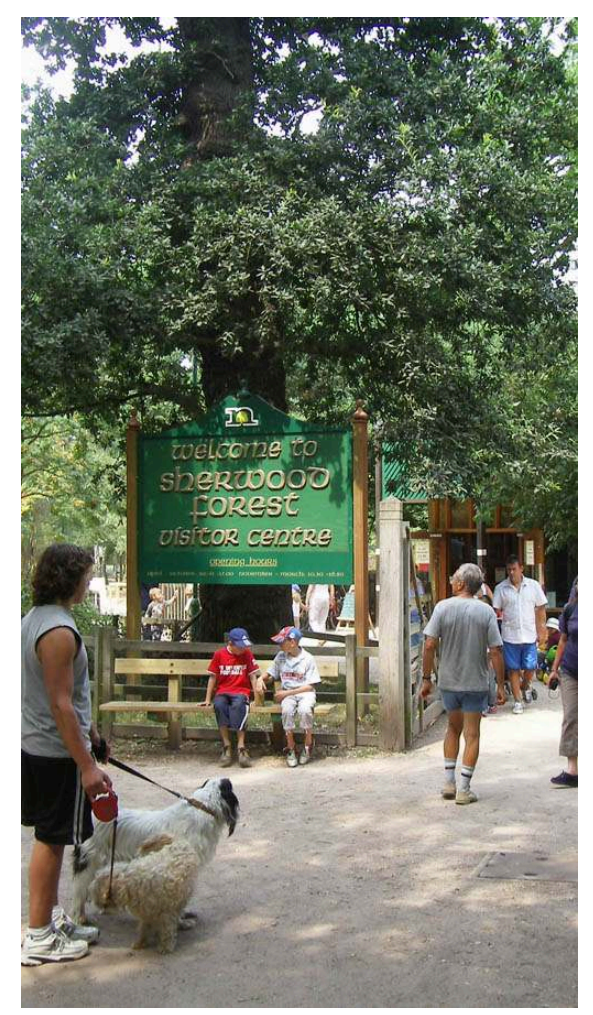

Les infrastructures, vétustes, concentrent les visiteurs sur une partie écologiquement et patrimonialement riche du massif ; aussi doivent-elles être reconstruites hors du massif proprement dit, entre la forêt et la petite ville voisine d'Edwinstowe.

42 L'accueil des 500000 visiteurs annuels est organisé autour d'un parking et d'un Visitor Centre (figure 5), et a pour attraction principale le Major Oak, l'arbre le plus connu de Grande-Bretagne : un chêne de plus de 800 ans, pesant 23 tonnes pour 10 mètres de circonférence : bien évidemment, Robin des Bois est censé l'avoir fréquenté. Réputé nationalement depuis le XIXe siècle, il a été soutenu dès les années 1900 par des 
chaînes, puis des structures en bois et des barres métalliques (figure 5). Il est protégé du piétinement par le public (source de compactage des sols) par une barrière en bois. Autour du Major Oak, s'étend la partie du massif la mieux préservée, les Birklands ; y subsistent en particulier plus de 900 arbres de plus de 500 ans, en particulier des chênes. Les peuplements sont dominés par les chênes locaux : chêne sessile (Quercus petraea), chêne pédonculé ( $Q$. robur); on y trouve un cortège feuillu classique de bouleaux (Betulus sp ;), sorbiers des oiseleurs (Sorbus aucuparia), houx.

Mais la plus grande partie de la forêt ne répond absolument pas à l'idée que l'on se fait de la mythique forêt de Robin et de ses compagnons. Elle porte la marque de la foresterie intensive du XXe siècle; une large opération de restauration a donc été engagée à travers une suite de projets coordonnés.

Dans les Birklands et autres espaces restés feuillus, la nouvelle gestion mise en place consiste essentiellement à restaurer une forêt largement ouverte, marquée par le pâturage, à la fois dans une optique écologique (restauration d'un habitat ouvert biodiversifié d'importance européenne) et culturelle (restauration des paysages considérés comme traditionnels). Les landes existantes doivent être protégées, et étendues ${ }^{7}$. Après une première tentative de restauration du pâturage qui s'est soldée par un échec (1998), en raison du couvert trop important formé par la fougère aigle (Pteridium aquilinum) inappétente, ovins et bovins ont désormais à nouveau leur place dans la forêt. L'intérêt économique direct du retour du pâturage est évidemment nul et, au contraire, l'opération est subventionnée.

Les changements de gestion sont particulièrement brusques dans les plantations voisines, où domine le pin laricio. Ici, il s'agit de maintenir une gestion productive tout en convertissant progressivement les peuplements vers un paysage plus conforme aux attentes du public, c'est-à-dire à des massifs feuillus ponctués d'ancient trees, voire à des wood-pastures. Une gestion fine et suivie scientifiquement a donc été mise en place. Le remplacement des plantations résineuses se fait progressivement, par ouverture de clairières dont les limites ne sont jamais en ligne droite. En outre, une attention toute particulière est portée aux ancient trees qui subsistent au sein des plantations. En effet, les opérations d'enrésinement ont épargné les plus vieux chênes, dont le bûcheronnage eût été particulièrement long et coûteux ; les vieux pollards sont donc restés en place, progressivement surcimés et étouffés par leurs nouveaux voisins résineux. Or, il est très rapidement apparu qu'un dégagement trop rapide des vétérans leur était dommageable, voire fatal, en raison d'un trop brusque changement de luminosité et de microclimat. On les dégage donc progressivement, en ouvrant autour d'eux des clairières d'abord étroites, puis progressivement élargies par des coupes renouvelées tous les cinq ans. L'arbre reste dans un premier temps abrité du côté sud, puis se trouve ensuite progressivement libéré et, autour de lui, sont réimplantées les essences feuillues qui doivent, à terme, composer la totalité, ou la quasi-totalité du massif de Sherwood. Les figures 7 et 8 ci-dessous montrent l'aspect très particulier du massif alors qu'un réseau de petites clairières commence à être ouvert autour des vieux chênes dans les parties enrésinées du massif, chaque clairière ayant une forme arrondie, comme si la ligne droite, trop associée aux épisodes de plantation et de gestion intensive, était désormais bannie du paysage forestier. 
Figure 4 : le massif de Sherwood / Thoresby. Sources : Ordnance Survey, images NAVTEQ, observations personnelles 2006.

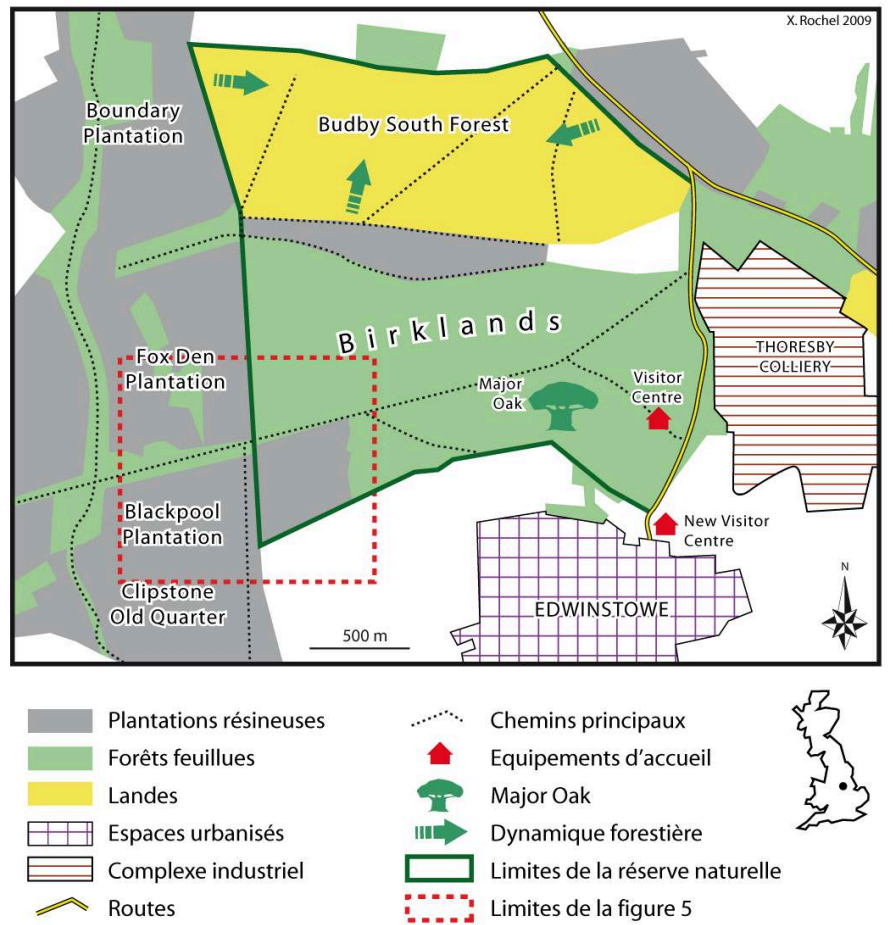

Les peuplements préservés des environs du Visitor Centre masquent mal un massif très largement enrésiné, même si des bandes feuillues ont parfois été préservées de part et d'autre des chemins principaux lors des plantations successives du XXe siècle.

Figure 5 : ouverture progressive des peuplements dans les environs du Centre Tree, Sherwood. Sources : Ordnance Survey, images NAVTEQ (2007) / Google Maps (2009).

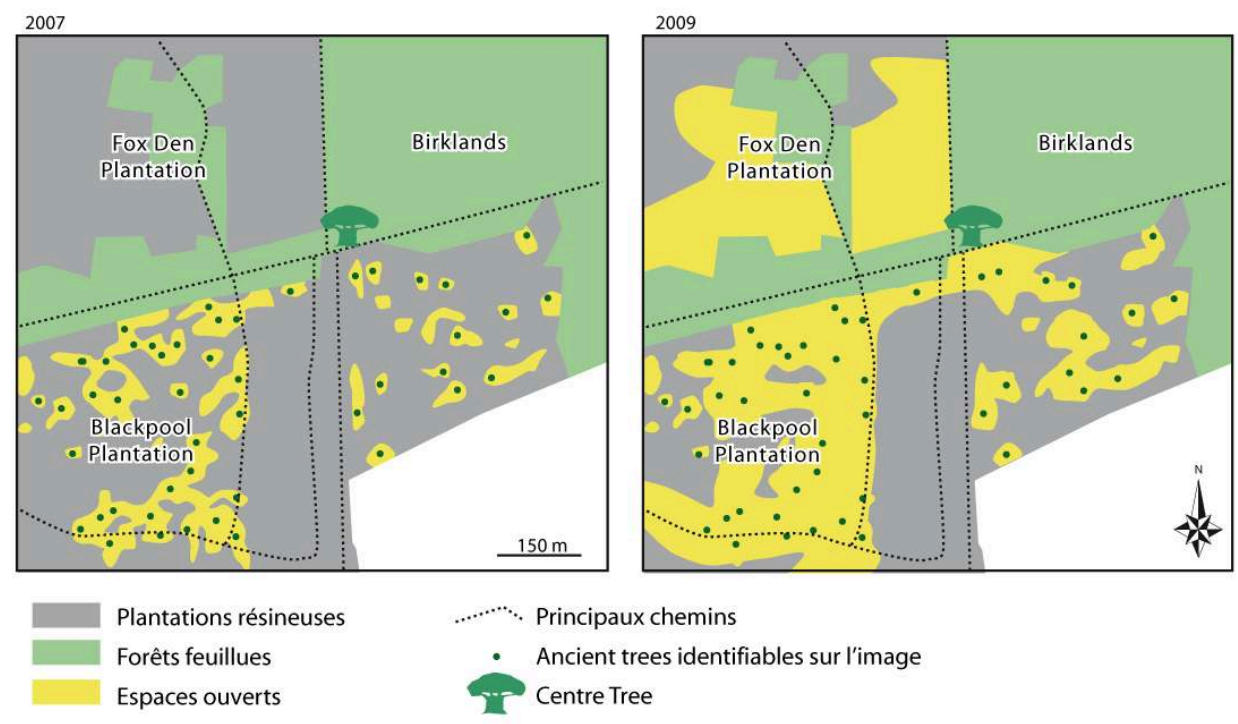




\section{Conclusion : quels modèles pour une nouvelle gestion forestière?}

Les sites ici explorés sont des sites extraordinaires dont la gestion ne saurait être ordinaire, et dont la valeur patrimoniale et culturelle justifie de laisser pour partie de côté la fonction de production. Ni Sherwood, ni Hatfield, ni Glenmore ne constituent le modèle parfait pour les massifs concernés par les nouvelles évolutions de la gestion forestière. On aurait pu se pencher sur d'autres sites : la forêt de Dean, la New Forest, Ashdown. Quoi qu'il en soit, il est évident que les années 1980 et surtout 1990 ont vu un changement majeur dans les mentalités, et dans les pratiques effectivement mises en œuvre sur le terrain; les forêts britanniques sont désormais intégrées au champ patrimonial, et ce changement majeur de fonction s'est traduit par ce qu'on peut assimiler à des conversions, voire des reconversions. Ce sont d'abord des considérations environnementales qui ont conduit aux opérations de retour vers un état antérieur des peuplements forestiers. Elles ont été, plus récemment, complétées par des considérations patrimoniales et esthétiques, non sans contradictions parfois majeures entre les attentes des uns et des autres : une gestion en faveur de l'équilibre écologique se concilie difficilement avec une gestion dite patrimoniale orientée vers le maintien des paysages "traditionnels » très anthropisés, de même que cette gestion patrimoniale ne peut complètement satisfaire ceux qui voient dans les forêts un espace de réserve laissé à lui-même.

Le modèle anglais, adapté à une certaine vision des espaces forestiers propre aux îles britanniques, n'est certainement pas un modèle applicable sur le continent. Il implique une gestion fine des peuplements qui confine à ce qu'est l'entretien des parcs urbains ou marmentaux; il est évidemment coûteux. Ceci convient dans un pays où les ancient woodlands, rares, vénérés, représentent à la fois un enjeu économique modeste et un enjeu culturel exceptionnel. Une telle attitude n'est envisageable en France qu'à l'échelle de quelques massifs et les massifs de Paimpont-»Brocéliande » et Fontainebleau, par exemple, surgissent immédiatement à l'esprit. Il reste que sans nier ni même contraindre fortement la fonction productrice de la forêt française, et des forêts européennes en général, une attitude respectueuse et raisonnée face au patrimoine culturel que représentent les forêts reste possible, comme l'atteste le projet «forêt-patrimoine" dans lequel s'est lancé l'office National des Forêts depuis 2007. Cette idée devrait légitimement prendre davantage de place dans les systèmes de certification censés mesurer, et reconnaître le caractère durable de la gestion forestière.

\section{BIBLIOGRAPHIE}

Agnoletti M. (2000). - “The Development of Forest History Research”. In : Agnoletti M., Anderson S. - Dir, Methods and Approaches in Forest History, Wallingford, New York, CABI Publishing, p. 1-20. 
Agnoletti M. - Dir. (2006). - The conservation of cultural landscapes, Wallingford, CABI publishing, $320 \mathrm{p}$.

Arnould P., Hotyat M., Simon L. (1997). — Les forêts d'Europe, Paris, Nathan, 413 p.

Bayfield N.G., Fraser N.M., Calle Z. (1998). - "High altitude colonisation of the Northern Corries of Cairn Gorm by Scots pine (Pinus sylvestris)", Scottish Geographical Magazine, 114-3, p. 172-179.

Cianco O., Nocentini S. (2000). - "Forest Management from positivism to the culture of complexity". In : Agnoletti M., Anderson S. - éd., Methods and Approaches in Forest History, CABI publishing, IUFRO Series $n^{\circ} 3$, p. 47-58.

Darby H.C. (1977). - Domesday England, Cambridge, New York, Cambridge University Press, 416 p. Dupouey J.-L., Dambrine E., Dardignac C., Georges-Leroy M. - éd. (2007). - La mémoire des forêts, Actes du colloque « forêt, archéologie et environnement », Nancy 2004, ONF / INRA, DRAC Lorraine, $294 \mathrm{p}$.

Egan P (1850. - Robin Hood and Little John: or the merry men of Sherwood forest, Johnson, 280 p. Galochet M., Rochel X. (2008). - « Les forêts face au développement durable ». In : Wackermann G. - dir., Le développement durable, Paris, Ellipses, p. 217-235.

Girel J. (2006). - «Quand le passé éclaire le présent : écologie et histoire du paysage », Géocarrefour, vol. 81, 4-2006, p. 249-264.

Hall M. (2000). - “Comparing Damages : Italian and American Concepts of Restoration”. In : Agnoletti M., Anderson S. - éd., Methods and Approaches in Forest History, Wallingford, New York, CABI Publishing, p. 165-172.

Harris E., Harris J., James N.D.G. (2003). - Oak : a British History. Bollington : Windgather Press, 241 p.

Hoskins W.G. (1955). - The Making of the English Landscape,Londres, Hodder \& Stoughton, 240 p.

Humphrey J. et al. (2003). - The restoration of wooded landscapes, Actes du colloque d'

Edinburg, 2000, Edinburg, Forestry Commission, 158 p.

Johann E, Rochel X. et al. (2004). - "History of secondary spruce forests: motives and spatial distribution in the past”. In : Speicker H. et al. - éd., Norway Spruce Conversion : Options and Consequences, Brill Leiden-Boston, p. 25-62.

Kottler D., Watkins Ch., Lavers C. (2005). - "The Transformation of Sherwood Forest in the Twentieth Century : the Role of Private Estate Forestry", Rural History, 16, p. 95-110.

Muir R. (2005). - Ancient Trees, Living Landscapes, Stroud, Tempus Publishing, 256 p.

Neville-Havins P.J. (1976). - The Forests of England, Londres, Robert Hale, 208 p.

Oosthoek J.-W. (2000). - “The logics of British Forest Policy, 1919-1970”. In : Proceedings of the 3rd Biennal Conference of the European Society for Ecological Economics, Vienne, Wirtschafts Universität, CD-ROM.

Peterken G.F. (1996). - Natural Woodland : Ecology and Conservation in Northern Temperate Regions, Cambridge University Press, $536 \mathrm{p}$.

Petit S., Watkins Ch. (2004). - « Pratiques paysannes oubliées : l'étêtage et l'émondage des arbres en Grande-Bretagne (1600-1900) », Études rurales, jan-juin 2004, p. 197-214.

Rackham O. (1976). - Trees and Woodland in the British Landscape, Londres, Dent, $234 \mathrm{p}$. 
Rackham O. (1986). - The History of the Countryside, Londres, Dent, $462 \mathrm{p}$.

Rackham O. (1989). - The Last Forest : the Story of Hatfield Forest, Londres, Dent, 302 p.

Rochel X. (2006). - "Les anciennes fonctions forestières : conflits, compromis et partages ». In :

Galochet M. - dir., La forêt, ressource et patrimoine, Paris, Ellipses,p. 155-169.

Rotherham I.D. (2006). - “Working landscapes or recreational showcases - sustainable forest management and the implications of cultural knowledge loss". In : Parrotta J. Agnoletti M., Johann E. - éd., Cultural Heritage and sustainable forest management : the role of traditional knowledge, Varsovie, MCPFE, p. 208-216.

Schama S. (1995). - Landscape and Memory, New York, Alfred A. Knopf, 652 p.

Short B. (2004). - "Environmental politics, custom and personal testimony: memory and lifespace on the late Victorian Ashdown Forest, Sussex", Journal of Historical geography, 30, p. $470-495$.

Smithers R. - éd. (2002). - Restoring Planted Ancient Woodland Sites, Londres, Woodland Trust, British Ecological Society, $34 \mathrm{p}$.

Smout C. - éd. (2003). - People and Woods in Scotland, Edinbourg, Edinburgh University Press, $244 \mathrm{p}$.

Thompson R., Humphrey J., Harmer R., Ferris R. (2003). - Restoration of Native Woodland on Ancient Woodland Site, Edinburg, Forestry Commission, $52 \mathrm{p}$.

Watkins Ch. - éd. (1998). - European Woods and Forests: Studies in Cultural History, Wallingford, CABI, 256 p.

White J. (1997). - “What is a veteran tree and where are they all?", Quarterly Journal of Forestry, 91-3, p. 222-226.

Williams M. (2000). - "Putting « flesh on the carbon-based bones » of forest history". In : Agnoletti M., Anderson S. - éd., Methods and Approaches in Forest History, CABI publishing, IUFRO Series $n^{\circ} 3$, p. 35-46.

Williams M. (2006). - Deforesting the Earth. From Prehistory to global crisis, Chicago, London, The University of Chicago Press, $543 \mathrm{p}$.

\section{NOTES}

1. Sur le contexte américain, voir: MACQUILLAN A.G. 1993. Cabbages and Kings: the Ethics and Aesthetics of New Forestry. Environmental Values, 2-3, p. 191-221.

2. Ministerial Conference on the Protection of Forests in Europe: organisation intergouvernementale chargée de coordonner l'expertise sur la gestion durable des forêts à l'échelle européenne.

3. On désigne ainsi les espaces qui, selon les archives, ont été boisés au moins depuis l'an 1600, considérant que les plantations en milieux ouverts ne sont apparues qu'au XVIIe siècle et ne se sont vraiment développées que dans les siècles ultérieurs. Cette ancienneté de l'état boisé est un gage de richesse ou, du moins, de forte valeur écologique. Les évolutions récentes de la recherche conduisent à relativiser la valeur de ce concept.

4. Le terme anglais a été privilégié dans les pages qui suivent, parce que le mot «parc», trop ambigu, semble impliquer l'existence d'une clôture, et que l'expression " pré-bois ", qui eût pu convenir, n'est généralement utilisée en France que dans le contexte montagnard.

5. BROWN Jonathan. Oh, bother! Nimbies do Battle with Council over Poohs Forest. The Independant, 21 avril 2007. 
6. Il faut noter que les puristes, suivant la tradition médiévale, situent la légende de Robin Hood à Barnsdale (Yorkshire), et non à Sherwood (Nottinghamshire).

7. La réserve naturelle centrée sur les Birklands vient de s'étendre en 2007 aux landes de Budby, immédiatement au Nord, portant la surface protégée de 220 à 423 ha.

\section{RÉSUMÉS}

Les changements d'ambition de la foresterie européenne se traduisent sur le terrain par des opérations dites de "restauration " ou de «conversion» des massifs forestiers exagérément anthropisés au cours des deux derniers siècles. Cet article présente, à travers trois exemples britanniques, les formes que peuvent prendre ces nouvelles orientations lorsque l'approche culturelle et paysagère vient compléter les préoccupations traditionnelles de la foresterie. Dans les forêts de Hatfield, près de Londres, de Glenmore, dans les Highlands d'Ecosse, et de Sherwood dans le Nottinghamshire, s'expérimente une façon renouvelée de faire cohabiter les différentes fonctions de l'espace forestier.

Changing ambitions in forestry lead to restoration operations in many anthropized forests in Europe. This paper presents the way this new approach is led in several British forests, where cultural and landscape concerns complete the traditional purposes of forestry. In the forests of Hatfield, near London, Glenmore, in the Scottish Highlands, and Sherwood in Nottinghamshire are experimented new ways of bringing together the different functions of woodlands in our societies.

Der Absichtswandel der europäischen Forstwirtschaft führt auf dem Gelände zu Restaurierungsund Wechselsmaßnahmen in den im Lauf der beiden letzten Jahrhunderte vom Menschen zu stark geprägten Wäldern. Mit Hilfe drei britischen Beispielen stellt dieser Artikel die Formen dieser Neuorientierung dar, wenn die Kultur- und Landschaftsbetrachtungsweise die traditionnelle Beschäftigung der Forstwirtschaft. In den Wäldern von Hatfield in der Nähe von London, von Glenmore in den schottischen Highlands und von Sherwood in dem Nottinghamshire wird eine neue Weise die unterschiedlichen Funktionen des Waldraumes probiert.

INDEX

Keywords : conversion, Forest, heritage, nature, restoration

Schlüsselwörter : Erbe, Natur, Restaurierung, Wald, Wechsel

Mots-clés : conversion, Forêt, nature, patrimoine, restauration

\section{AUTEUR}

\section{XAVIER ROCHEL}

Maître de Conférences, Université Nancy 2. 3 place Godefroi de Bouillon, B.P. 331754015 NANCY Cedex. 